\title{
Urban-rural differences in adolescent eating behaviour: a multilevel cross-sectional study of 15-year-olds in Scotland
}

\author{
Kate A Levin ${ }^{1,2, *}$ \\ ${ }^{1}$ Child and Adolescent Health Research Unit, University of St Andrews, Medical and Biological Sciences \\ Building, North Haugh, St Andrews KY16 9TF, UK: ' 2 Ludwig Boltzmann Institute for Health Promotion Research, \\ Untere Donaustrasse 47, A-1020 Vienna, Austria
}

Submitted 8 February 2013: Final revision received 25 May 2013: Accepted 13 June 2013: First published online 21 August 2013

\begin{abstract}
Objective: Improving the diet of the Scottish population has been a government focus in recent years. Population health is known to vary between geographies; therefore alongside trends and socio-economic inequalities in eating behaviour, geographic differences should also be monitored.

Design: Eating behaviour data from the 2010 Scotland Health Behaviour in School-aged Children survey were modelled using multilevel linear and logistic modelling.

Setting: Data were collected in schools across urban and rural Scotland.

Subjects: Schoolchildren aged 15 years.

Results: Adolescents living in remote rural Scotland had the highest consumption frequency of vegetables (on average consumed on $6.68 \mathrm{~d} /$ week) and the lowest consumption frequency of sweets and crisps (on $4 \cdot 27$ and $3 \cdot 02 \mathrm{~d} /$ week, respectively). However, it was not in the major four cities of Scotland (Glasgow, Edinburgh, Dundee and Aberdeen) but in the geography described by the classification 'other urban' areas (large towns of between 10000 and 125000 residents) that adolescents had the poorest diet. Deprivation and rurality were independently associated with food consumption for all but fruit consumption. Sharing a family meal, dieting behaviour, food poverty and breakfast consumption did not differ by rurality. Variance at the school level was significant for fruit and vegetable consumption frequencies and for irregular breakfast consumption, regardless of rurality.

Conclusions: Young people from rural areas have a healthier diet than those living in urban areas. The eating behaviours examined did not explain these differences. Future research should investigate why urban-rural differences exist for consumption frequencies of 'healthy' and 'unhealthy' foods.
\end{abstract}

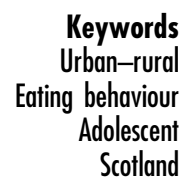

The Scottish Government, in accordance with international guidelines such as the WHO Global Strategy on Diet, Physical Activity and Health ${ }^{(1)}$ and the WHO 2008-2013 Action Plan for the Global Strategy for the Prevention and Control of Noncommunicable Diseases ${ }^{(2)}$, has in recent years prioritised improving the diet of the Scottish population by increasing healthy eating and reducing unhealthy eating $^{(3-5)}$. As with many health outcomes, however, some members of the population fare better than others. Within Scotland, gender and age differences have been shown in eating behaviour, with fruit and vegetable consumption more prevalent among girls and younger children, and sweets, chips and crisps consumption less prevalent ${ }^{(6)}$. Socio-economic inequalities in health have also been shown for many adolescent outcomes in Scotland and have widened over time for both adult ${ }^{(7,8)}$ and adolescent health measures ${ }^{(9,10)}$. A recent study of adolescent eating behaviour showed that socio-economic inequalities in adolescent eating habits exist in Scotland, with those from higher social class reporting a more favourable diet ${ }^{(11)}$.

Scotland has a population of approximately 5250000 with a land mass of $78772 \mathrm{~km}^{2}$, resulting in a relatively low average population density of sixty-five people per square kilometre. However, Scotland is highly urbanised, with most of the population residing in the central belt which includes the two largest cities, Glasgow and Edinburgh, and several other large towns. The Highlands and Islands, home to $7 \%$ of the Scottish population, makes up over $60 \%$ of Scottish land mass, with a resulting sparse population density of eight people per square kilometre. The heterogeneous nature of rural communities both in Scotland and the rest of the UK has been discussed in previous studies ${ }^{(12,13)}$, with health outcomes suggesting large variation in need within rural Scotland.

Levin et al. ${ }^{(11)}$ noted that eating behaviour differed by education authority, a large-area level with population 
size ranging between approximately 50000 and 550000 , with those from the Highlands and Islands having a healthier diet. Previous research on adult health has shown some geographical differences, with generally less favourable outcomes in remote rural Scotland: higher rates of suicide ${ }^{(14)}$ and IHD following discharge from hospital $^{(15)}$, more severe injuries due to road traffic accidents ${ }^{(16)}$ and more advanced stages of cancer at diagnosis $^{(17)}$. Theories around the unique hazards faced by rural residents from both the physical and built rural environment, including issues of access and availability of health services, exposure to health risks, as well as cultural differences influencing attitudes and behaviours and their impact on the health and well-being of the remote and rural population, are discussed elsewhere ${ }^{(18,19)}$. The studies of urban-rural inequalities in adult health listed above adjusted for socio-economic status using small-area level indicators of deprivation. Adjustment for deprivation is advisable in such studies as some geographies have higher rates of deprivation than others. For example, accessible rural Scotland is a relatively affluent geography ${ }^{(20)}$. Deprivation may therefore confound any association linking health and place. Adjustment for area-level deprivation should overcome confounding of this type.

Studies of inequalities, and particularly socio-economic inequalities, in health are widely documented. These are important, in part because as health improves, as it has done over recent decades in Scotland, greater improvements are generally observed among some members of the population than others ${ }^{(21,22)}$, but also because by identifying subgroups within the population whose health is particularly poor or particularly good, we may progress to identify associated modifiable risk factors. Studies of urban-rural inequalities in health, and particularly child health, in Scotland are limited, although geographic differences in health in Scotland are known to be larger than in any other part of the $\mathrm{UK}^{(23)}$, and this was raised as an area of inequalities requiring further work in the Inequalities in Health Report ${ }^{(24)}$. We are not aware of any previous study, however, that has investigated urbanrural differences in eating behaviour or indeed any health outcome in adolescence. The present study aimed to describe patterns of eating behaviour among adolescents across the urban-rural spectrum, after adjustment for individuals' family affluence and area-level deprivation.

\section{Methods}

\section{Study design}

The study examines Scottish data from the 2010 Health Behaviour in School-aged Children (HBSC) survey. HBSC is a WHO Collaborative Cross-National Study conducted in countries across Europe and North America and uses a standardised protocol ${ }^{(25)}$. For the HBSC Scotland survey, the population was stratified by education authority and school type, defined as state-funded or independent, and a nationally representative sample was selected using systematic random sampling. Using passive parental consent except where active consent was required, pupils in Secondary 4 (S4), aged approximately 15.5 years, received questionnaires in school between January and March. The questionnaire was completed anonymously in class under teacher supervision. The research protocol was approved by the University of Edinburgh's School of Education Ethics Committee.

The 2010 HBSC Scotland survey sample of S4 pupils, aged on average 15.5 years, was boosted to give a representative sample of each of the six ruralities defined by the Scottish Household Survey and described in Table 1. Urban samples are generally well represented within the survey as they make up a large part of the population. However, rural samples, and particularly the remote rural sample, are often small as they make up only a small proportion of the population and the HBSC sample is selected to be representative of Scotland as a whole. The boosted sample of classes was selected randomly within each sampling frame, defined by rurality classification, assigned by school postcode. The samples were boosted with the aim of achieving a minimum of 350 children within each rurality classification to give a $95 \%$ confidence interval of $\pm 6 \%$ around a proportion of $65 \%$ (for 15 -year-olds, the majority

Table 1 Definition of the urban-rural classification used

\begin{tabular}{|c|c|c|c|}
\hline Rural classification & Description* & $\begin{array}{l}\% \text { of study } \\
\text { sample }\end{array}$ & $\begin{array}{l}\% \text { of Scottish } \\
\text { population* }\end{array}$ \\
\hline Four Cities & $\begin{array}{l}\text { Settlements with population over } 125000 \text { (i.e. Aberdeen, Dundee, Glasgow } \\
\text { and Edinburgh) }\end{array}$ & $23 \cdot 8$ & $38 \cdot 9$ \\
\hline Other Urban & Other settlements with population over 10000 & $23 \cdot 2$ & $30 \cdot 3$ \\
\hline Accessible Towns & $\begin{array}{l}\text { Settlements with population between } 3000 \text { and } 10000 \text { and within a } 30 \text {-min drive } \\
\text { of a settlement with population } 10000 \text { or more }\end{array}$ & $10 \cdot 7$ & $8 \cdot 6$ \\
\hline Remote Towns & $\begin{array}{l}\text { Settlements with population between } 3000 \text { and } 10000 \text { and more than a } 30 \text {-min drive } \\
\text { to a settlement with population } 10000 \text { or more }\end{array}$ & $15 \cdot 7$ & $4 \cdot 1$ \\
\hline Accessible Rural & $\begin{array}{l}\text { Settlements with population less than } 3000 \text { and within a } 30-\text { min drive of a settlement } \\
\text { with population } 10000 \text { or more }\end{array}$ & $8 \cdot 8$ & $11 \cdot 2$ \\
\hline Remote Rural & $\begin{array}{l}\text { Settlements with population less than } 3000 \text { and more than a } 30 \text {-min drive to a } \\
\text { settlement with population } 10000 \text { or more }\end{array}$ & $17 \cdot 7$ & $7 \cdot 0$ \\
\hline
\end{tabular}

*Scottish Government $(2008)^{(35)}$ 
of variables saw proportions greater than $65 \%$ or smaller than $35 \%$ in the 2006 HBSC survey ${ }^{(26)}$ ) and a design factor of $1 \cdot 2$. The response rate of the boosted rural sample was high, higher than the rest of Scotland, probably due to the fact that schools in remote and rural areas are not called upon as often as their urban counterparts to take part in research surveys.

\section{Outcome variables}

Consumption of fruit, vegetables, sweets, crisps and chips were examined in the study with the question 'How many times a week do you usually eat the following things?' and response options of 'never', 'less than once a week', 'once a week', '2-4 days a week', '5-6 days a week', 'once a day, every day' and 'every day more than once'. This measure has been validated previously among Belgian adolescents $^{(27)}$.

The seven optional responses for each question were re-coded as follows: 'never' $=0$; 'less than once a week' $=0 \cdot 25$ (equivalent to consumption of food item once every 4 weeks); 'once a week' $=1$; '2-4 days a week' $=3$; '5-6 days a week' $=5 \cdot 5$; and 'once a day, every day'/'every day more than once' $=7$. This represents the number of days per week each food item is consumed. Recoding the food items in this way has been previously validated $^{(28,29)}$. A further composite score of 'healthy eating' combined all five items by adding weekly vegetable and fruit consumption and subtracting consumption of sweets, chips and crisps, with coding as above except for response 'more than once day' $=14$, a method used previously in the construction of food indices ${ }^{(11,29,30)}$. This was centred to give a score ranging between $-42 \cdot 0$ and $28 \cdot 0$, with a mean of 0 . The measure had a kurtosis of 0.63 and skewness of $-0 \cdot 27$, acceptable for linear analyses. Similarly, all other outcomes had a kurtosis of $<1.34$ and a skewness of $<0 \cdot 83$.

A further set of eating behaviour measures was examined using binary outcomes: eating a family meal together four or more times per week; regular breakfast consumption (every day during the school week); on a diet to reduce weight; and going to bed hungry, also known as 'food poverty', an indicator of a disordered household ${ }^{(31)}$. Descriptions of these four survey questions and optional responses, as well as reports of prevalence nationally and internationally, are available elsewhere ${ }^{(6,31,32)}$.

\section{Explanatory variables}

Young people's age and sex were included in analysis. School type (state or independent) was also included. The Family Affluence Scale (FAS) ${ }^{(33)}$ was calculated using responses to the following questions: 'Does your family have a car or van?' (response options: 'no'/'yes, one'/'yes, two or more'); 'Do you have your own bedroom to yourself?' (response options: 'no'/'yes'); 'During the past 12 months, how many times did you travel away on holiday with your family?' (response options: 'not at all'/'once'/"twice or more'); and 'How many computers (PCs, Macs or laptops) does your family own?' (response options: 'none'/'one'/'two'/'more than two'). The items were combined using categorical principal components analysis to produce tertiles of low, medium and high family affluence, as recommended ${ }^{(33)}$.

Deprivation at the area level was also included using the 2006 Scottish Index of Multiple Deprivation (SIMD), a continuous measure of deprivation at the 'data zone' small-area level which combines thirty-seven indicators across seven domains: income, employment, health, education, housing, geographic access and crime ${ }^{(34)}$. The 2006 SIMD score included in the present analysis ranged from 1.04 (least deprived) to $85 \cdot 85$ (most deprived), assigned to each child by his/her home postcode. The results presented were for a unit change in deprivation as measured by the SIMD score divided by ten, with the assumption that each additional unit carries an equivalent effect. Rurality was included as a categorical variable as defined by the 2008 Scottish Household Survey urban-rural classification ${ }^{(35)}$ and used in similar analyses elsewhere ${ }^{(13-15,36)}$.

Of the original 3577 young people surveyed, 894 (25\%) were excluded due to missing postcode information, $54 \%$ boys and $46 \%$ girls. The final data set had 2683. Among those excluded, fruit and vegetable consumption was slightly lower than for those included in the study, and consumption of chips, sweets and chips was slightly higher. However, there did not appear to be any response bias by affluence, with $34 \%$ of those excluded having low FAS, 34\% middle FAS and $32 \%$ high FAS. A further twenty-four $(0 \cdot 9 \%)$, thirty-two $(1 \cdot 2 \%)$, twenty-nine $(1 \cdot 1 \%)$, thirty-eight $(1 \cdot 4 \%)$ and twenty-four $(0 \cdot 9 \%)$ had missing fruit, vegetable, sweets, crisps and chips consumption information and therefore had to be excluded from analyses for individual items, with ninety-three (3.5\%) missing a healthy eating score.

\section{Statistical analysis}

Preliminary analyses described the data, presenting prevalence of eating behaviour and mean number of days foods were consumed. These were compared using $t$ tests (for means) and $\chi^{2}$ tests (for prevalence) to assess patterns of urban-rural inequalities in outcomes as a preliminary analysis. Linear multilevel regression models were then fitted for each of the five scale outcome variables and combined healthy eating variable, using RIGLS (restricted iterative generalised least squares) estimation in the statistical package MLwiN $2 \cdot 02^{(37)}$. Fixed and random parameter estimates were tabulated. Joint $\chi^{2}$ tests were carried out to determine the significance of variables and Wald tests to identify the significance of parameter estimates. The Akaike Information Criterion (AIC) was used as a measure of model fit with a lower AIC value being favoured. The models had three levels: education authority, school and individual child. The models were fitted, adjusting for age, sex, school type (state or 
independent), FAS, SIMD score/10 and rurality, to describe differences by geography. Parameter estimates were tabulated and discussed. Consumption frequency variables and the healthy eating score were treated as normally distributed in the analyses. Residuals of the models and the results of binary models of consumption with outcome 'high' $v$. 'low' consumption, as defined elsewhere ${ }^{(28)}$, support this modelling structure and the findings presented here, and are available from the author on request.

Binary eating behaviour outcomes were modelled using the MCMC (Markov chain Monte Carlo) method in MLwiN and fixed and random parameter estimates were tabulated. Wald tests were carried out to identify the significance of parameter estimates. Estimates reported in the results are based on a chain of length of 50000 following a burn-in of 5000. The Deviance Information Criterion (DIC) was used as a measure of model fit with a lower value of the DIC being favoured ${ }^{(38)}$.

\section{Results}

Table 2 describes consumption frequency and eating behaviour by geography. Mean number of days where fruit was consumed was particularly low in Other Urban areas. Vegetables were also consumed on fewer days in more urban areas, while Remote Rural and Accessible Rural areas saw the lowest consumption frequencies of sweets, chips and crisps; e.g. crisps were consumed on average approximately $4 \mathrm{~d}$ /week in Four Cities, compared with $3 \mathrm{~d} /$ week in Remote Rural areas. There were also some differences observed between Remote Towns and Remote Rural areas; young people living in Remote Towns consumed vegetables on fewer days and crisps on more days than those living in Remote Rural areas. The overall healthy eating score was particularly high $(3 \cdot 11)$ in Remote Rural areas and lowest in Other Urban areas $(-2 \cdot 42)$. While consumption frequency differed by rurality, eating behaviours for the most part did not. Eating a family meal together on $\geq 4 \mathrm{~d} /$ week, however, was significantly more prevalent in Remote Towns (78\%) compared with Four Cities (67\%) at the $95 \%$ level of significance, while prevalence in Other Urban areas was also lower $(68 \%)$ at the $93 \%$ level of significance.

When the data were modelled, the healthy eating score was greater for girls (on average 2.92 more than for boys), due to greater frequency of fruit and vegetable consumption and lower frequency of sweets and chips consumption (Table 3). Children attending independent

Table 2 Consumption frequency and eating behaviour variables by rurality among 15-year-old adolescents; data from the 2010 Scotland Health Behaviour in School-aged Children survey

\begin{tabular}{|c|c|c|c|c|c|c|c|c|c|c|c|c|c|}
\hline \multirow[b]{2}{*}{ Categorical measures } & \multicolumn{2}{|c|}{$\begin{array}{l}\text { Four Cities } \\
\quad(n \text { 639) }\end{array}$} & \multicolumn{2}{|c|}{$\begin{array}{l}\text { Other Urban } \\
\quad(n \text { 623) }\end{array}$} & \multicolumn{2}{|c|}{$\begin{array}{c}\text { Accessible Towns } \\
(n \text { 288) }\end{array}$} & \multicolumn{2}{|c|}{$\begin{array}{l}\text { Remote Towns } \\
\quad(n \text { 235) }\end{array}$} & \multicolumn{2}{|c|}{$\begin{array}{l}\text { Accessible Rural } \\
\text { (n 422) }\end{array}$} & \multicolumn{2}{|c|}{$\begin{array}{l}\text { Remote Rural } \\
\qquad(n 476)\end{array}$} & \multirow[b]{2}{*}{$P$ value $^{\star}$} \\
\hline & $\%$ & $n$ & $\%$ & $n$ & $\%$ & $n$ & $\%$ & $n$ & $\%$ & $n$ & $\%$ & $n$ & \\
\hline \multicolumn{14}{|l|}{ Meal together } \\
\hline$\geq 4 \mathrm{~d} /$ week & $67 \cdot 4$ & 428 & $68 \cdot 2$ & 422 & $71 \cdot 6$ & 204 & $78 \cdot 0$ & 181 & $71 \cdot 3$ & 298 & $74 \cdot 1$ & 352 & \multirow{2}{*}{$\underset{\mathrm{a}}{0.015}$} \\
\hline$<4 \mathrm{~d} /$ week & $32 \cdot 6$ & 207 & $31 \cdot 8$ & 197 & $28 \cdot 4$ & 81 & $22 \cdot 0$ & 51 & $28 \cdot 7$ & 120 & $25 \cdot 9$ & 123 & \\
\hline \multicolumn{14}{|l|}{ Breakfast } \\
\hline Regular breakfast consumption & $56 \cdot 0$ & 357 & $54 \cdot 5$ & 338 & $52 \cdot 3$ & 150 & $53 \cdot 2$ & 124 & $60 \cdot 4$ & 255 & $54 \cdot 4$ & 259 & \multirow[t]{2}{*}{$0 \cdot 270$} \\
\hline Irregular breakfast consumption & $44 \cdot 0$ & 280 & $45 \cdot 5$ & 282 & $47 \cdot 7$ & 137 & $46 \cdot 8$ & 109 & $39 \cdot 6$ & 167 & $45 \cdot 6$ & 217 & \\
\hline \multicolumn{14}{|l|}{ On a diet } \\
\hline Not on a diet & $80 \cdot 8$ & 513 & $80 \cdot 2$ & 497 & $76 \cdot 8$ & 219 & $76 \cdot 1$ & 178 & $82 \cdot 4$ & 347 & $80 \cdot 3$ & 380 & \multirow[t]{2}{*}{$0 \cdot 319$} \\
\hline On a diet & $19 \cdot 2$ & 122 & $19 \cdot 8$ & 123 & $23 \cdot 2$ & 66 & $23 \cdot 9$ & 56 & $17 \cdot 6$ & 74 & $19 \cdot 7$ & 93 & \\
\hline \multicolumn{14}{|l|}{ Go to bed hungry } \\
\hline Rarely or never & 95.9 & 613 & $95 \cdot 2$ & 592 & $93 \cdot 0$ & 267 & $94 \cdot 4$ & 221 & $96 \cdot 0$ & 404 & $96 \cdot 0$ & 455 & \multirow[t]{2}{*}{$0 \cdot 390$} \\
\hline Sometimes or often & $4 \cdot 1$ & 26 & $4 \cdot 8$ & 30 & $7 \cdot 0$ & 20 & $5 \cdot 6$ & 13 & $4 \cdot 0$ & 17 & $4 \cdot 0$ & 19 & \\
\hline Continuous measures & Mean & SD & Mean & SD & Mean & SD & Mean & SD & Mean & SD & Mean & SD & \\
\hline Fruit consumptiont & $5 \cdot 40$ & $4 \cdot 65$ & $5 \cdot 06$ & $4 \cdot 44$ & $5 \cdot 05$ & $4 \cdot 69$ & $5 \cdot 30$ & $4 \cdot 65$ & $5 \cdot 78$ & 4.54 & $5 \cdot 63$ & $4 \cdot 40$ & 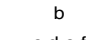 \\
\hline Vegetable consumptiont & $5 \cdot 72$ & 4.52 & $5 \cdot 61$ & $4 \cdot 31$ & $5 \cdot 51$ & $4 \cdot 32$ & $5 \cdot 82$ & $2 \cdot 65$ & $6 \cdot 48$ & $4 \cdot 37$ & $6 \cdot 68$ & $4 \cdot 42$ & $\mathrm{c}, \mathrm{d}, \mathrm{e}, \mathrm{f}$ \\
\hline Sweets consumptiont & $5 \cdot 57$ & $4 \cdot 01$ & $5 \cdot 65$ & $4 \cdot 10$ & $5 \cdot 31$ & $3 \cdot 96$ & 4.97 & $2 \cdot 30$ & $4 \cdot 71$ & 3.51 & $4 \cdot 27$ & $3 \cdot 20$ & $\mathrm{~g}, \mathrm{~h}, \mathrm{i}$ \\
\hline Crisps consumptiont & $4 \cdot 06$ & $3 \cdot 90$ & $4 \cdot 34$ & $3 \cdot 88$ & $3 \cdot 80$ & $3 \cdot 73$ & $3 \cdot 80$ & $2 \cdot 51$ & $3 \cdot 47$ & $3 \cdot 49$ & $3 \cdot 02$ & $3 \cdot 26$ & $c, e, f, j$ \\
\hline Chips consumptiont & $2 \cdot 46$ & $2 \cdot 63$ & $2 \cdot 99$ & $2 \cdot 97$ & $2 \cdot 50$ & $2 \cdot 82$ & $2 \cdot 36$ & $2 \cdot 44$ & $2 \cdot 23$ & $2 \cdot 45$ & $2 \cdot 01$ & $2 \cdot 04$ & $\mathrm{k}, \mathrm{l}$ \\
\hline Healthy eating scoreł & -0.85 & $12 \cdot 43$ & $-2 \cdot 42$ & $12 \cdot 26$ & -0.90 & $12 \cdot 08$ & $-0 \cdot 10$ & $10 \cdot 77$ & $1 \cdot 81$ & $11 \cdot 14$ & $3 \cdot 11$ & $10 \cdot 42$ & $\mathrm{~g}, \mathrm{j}, \mathrm{m}, \mathrm{n}$ \\
\hline
\end{tabular}

${ }^{a}$ Four Cities and Remote Towns differ significantly at $95 \%$ level of significance; ${ }^{b}$ Other Urban differs significantly from Remote Rural and Accessible Rural at $95 \%$ level of significance; ${ }^{c}$ Four Cities, Other Urban and Accessible Towns differ significantly from Remote Rural at $99 \%$ level of significance; ${ }^{d}$ Other Urban

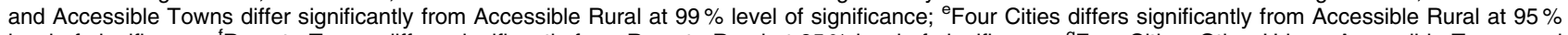

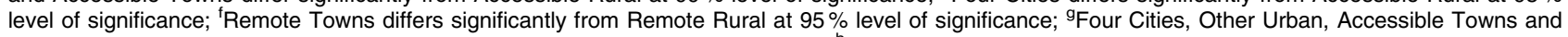
Remote Towns differ significantly from Remote Rural at $99 \%$ level of significance; ${ }^{\text {h}}$ Four Cities and Other Urban differ significantly from Accessible Rural at $99 \%$ level of significance; ' $F$ Four Cities and Other Urban differ significantly from Remote Towns at $95 \%$ level of significance; ${ }^{j}$ Other Urban differs significantly from Accessible Rural at $99 \%$ level of significance; ${ }^{k}$ Other Urban differs significantly from Remote Rural, Accessible Rural and Remote Towns at $99 \%$ level of significance; 'Remote Rural differs from Four Cities at $95 \%$ level of significance; ${ }^{m}$ Four Cities and Accessible Towns differ significantly from Accessible Rural at $95 \%$ level of significance; ${ }^{n}$ Other Urban differs significantly from Remote Towns at $95 \%$ level of significance.

${ }^{*} P$ value from $\chi^{2}$ test for categorical measures and from $t$ test for continuous measures.

tNumber of days per week the item is consumed.

‡Healthy weekly eating score calculated by adding weekly fruit and vegetable consumption and subtracting weekly crisps, sweets and chips consumption. 
Table 3 Multilevel linear models for eating behaviour outcomes (REML estimates*; sE) among 15-year-old adolescents; data from the 2010 Scotland Health Behaviour in School-aged Children survey

\begin{tabular}{|c|c|c|c|c|c|c|c|c|c|c|c|c|}
\hline & \multicolumn{2}{|c|}{ Fruit consumption } & \multicolumn{2}{|c|}{ Vegetable consumption } & \multicolumn{2}{|c|}{ Sweets consumption } & \multicolumn{2}{|c|}{ Crisps consumption } & \multicolumn{2}{|c|}{ Chips consumption } & \multicolumn{2}{|c|}{ Healthy eating score } \\
\hline & Mean & SE & Mean & SE & Mean & SE & Mean & SE & Mean & SE & Mean & SE \\
\hline \multicolumn{13}{|l|}{ Fixed effects } \\
\hline Constant/intercept & $5 \cdot 46$ & $2 \cdot 32$ & $1 \cdot 83$ & $2 \cdot 23$ & $5 \cdot 40$ & $2 \cdot 12$ & $7 \cdot 15$ & $2 \cdot 39$ & $1 \cdot 33$ & $1 \cdot 87$ & $-10 \cdot 23$ & $11 \cdot 05$ \\
\hline \multirow{2}{*}{\multicolumn{13}{|c|}{ Sex (ref.: Male) }} \\
\hline & & & & & & & & & & & & \\
\hline $\begin{array}{l}\text { Female } \\
\text { FAS (ref.: Low FAS) }\end{array}$ & $0 \cdot 42$ & $0 \cdot 09 \ddagger$ & $0 \cdot 31$ & $0.09 \ddagger$ & -0.33 & $0.09 \ddagger$ & -0.04 & $0 \cdot 10$ & $-0 \cdot 46$ & $0.08 \ddagger$ & $2 \cdot 92$ & $0 \cdot 45 \ddagger$ \\
\hline Medium FAS & $0 \cdot 11$ & $0 \cdot 12$ & 0.26 & $0 \cdot 11 \ddagger$ & -0.07 & $0 \cdot 11$ & $-0 \cdot 16$ & $0 \cdot 12$ & -0.33 & $0.09 \ddagger$ & $1 \cdot 19$ & $0.55 \ddagger$ \\
\hline High FAS & 0.23 & $0 \cdot 12$ & $0 \cdot 24$ & $0 \cdot 12 \ddagger$ & 0.04 & $0 \cdot 11$ & $-0 \cdot 14$ & $0 \cdot 12$ & $-0 \cdot 19$ & $0 \cdot 10 \ddagger$ & $1 \cdot 18$ & $0.57 \ddagger$ \\
\hline \multirow{2}{*}{\multicolumn{13}{|c|}{ School type (ref.: State school) }} \\
\hline & & & & & & & & & & & & \\
\hline Independent school & 0.94 & $0 \cdot 29 \ddagger$ & 0.99 & $0 \cdot 26 \ddagger$ & $-0 \cdot 12$ & 0.29 & $-0 \cdot 81$ & $0 \cdot 37 \ddagger$ & -0.77 & $0 \cdot 30 \ddagger$ & $5 \cdot 78$ & $1.53 \ddagger$ \\
\hline \multicolumn{13}{|l|}{ Rurality (ref.: Four Cities) } \\
\hline Other Urban & -0.20 & $0 \cdot 16$ & -0.01 & $0 \cdot 15$ & -0.05 & $0 \cdot 15$ & $0 \cdot 20$ & $0 \cdot 18$ & 0.47 & $0 \cdot 14 \ddagger$ & $-1 \cdot 73$ & $0 \cdot 81 \ddagger$ \\
\hline Accessible Towns & $-0 \cdot 42$ & $0 \cdot 20 \ddagger$ & $-0 \cdot 14$ & $0 \cdot 18$ & $-0 \cdot 20$ & $0 \cdot 18$ & $-0 \cdot 13$ & 0.21 & $0 \cdot 11$ & $0 \cdot 16$ & -0.90 & 0.98 \\
\hline Remote Towns & $-0 \cdot 13$ & $0 \cdot 21$ & $0 \cdot 16$ & $0 \cdot 19$ & -0.32 & $0 \cdot 19$ & $-0 \cdot 12$ & 0.23 & $0 \cdot 12$ & $0 \cdot 18$ & 0.22 & 1.06 \\
\hline Accessible Rural & 0.08 & $0 \cdot 17$ & $0 \cdot 31$ & $0 \cdot 16$ & -0.41 & $0 \cdot 16 \ddagger$ & -0.22 & $0 \cdot 18$ & $0 \cdot 11$ & $0 \cdot 14$ & $1 \cdot 02$ & 0.85 \\
\hline Remote Rural & $0 \cdot 13$ & $0 \cdot 17$ & 0.55 & $0 \cdot 16 \ddagger$ & -0.65 & $0 \cdot 16 \ddagger$ & -0.67 & $0 \cdot 19 \ddagger$ & -0.08 & $0 \cdot 15$ & $2 \cdot 90$ & $0.88 \ddagger$ \\
\hline \multicolumn{13}{|l|}{ Random effects } \\
\hline Level 1 (child) variance & $5 \cdot 645$ & $0 \cdot 160$ & $5 \cdot 266$ & $0 \cdot 149$ & $4 \cdot 759$ & $0 \cdot 135$ & $5 \cdot 979$ & $0 \cdot 169$ & $3 \cdot 662$ & $0 \cdot 103$ & $123 \cdot 100$ & 3.530 \\
\hline Level 2 (school) variance & $0 \cdot 173$ & 0.058 & 0.091 & 0.046 & 0.046 & 0.041 & 0.084 & 0.055 & 0.042 & 0.033 & $4 \cdot 836$ & 1.547 \\
\hline Level 3 (education authority) variance & 0.000 & 0.000 & 0.000 & 0.000 & 0.026 & 0.024 & 0.058 & 0.039 & 0.042 & 0.025 & 0.293 & 0.687 \\
\hline-2 log likelihood & \multirow{2}{*}{\multicolumn{2}{|c|}{$\begin{array}{c}12200 \cdot 8 \\
15\end{array}$}} & \multicolumn{2}{|c|}{$11955 \cdot 1$} & \multicolumn{2}{|c|}{$11693 \cdot 6$} & \multicolumn{2}{|c|}{$12272 \cdot 0$} & \multicolumn{2}{|c|}{$11030 \cdot 4$} & \multicolumn{2}{|c|}{$19885 \cdot 5$} \\
\hline No. of parameters & & & \multirow{2}{*}{\multicolumn{2}{|c|}{$\begin{array}{c}15 \\
11985 \cdot 1\end{array}$}} & \multirow{2}{*}{\multicolumn{2}{|c|}{$\begin{array}{c}15 \\
11723 \cdot 6\end{array}$}} & \multirow{2}{*}{\multicolumn{2}{|c|}{$\begin{array}{c}15 \\
12302 \cdot 0\end{array}$}} & \multirow{2}{*}{\multicolumn{2}{|c|}{$\begin{array}{c}15 \\
11060 \cdot 4\end{array}$}} & \multicolumn{2}{|c|}{$\begin{array}{c}15 \\
19015.5\end{array}$} \\
\hline $\mathrm{AIC}+$ & \multicolumn{2}{|c|}{$12230 \cdot 8$} & & & & & & & & & $19 \varsigma$ & \\
\hline AIC+ for model without rurality & \multicolumn{2}{|c|}{$12231 \cdot 7$} & \multicolumn{2}{|c|}{$11996 \cdot 3$} & \multicolumn{2}{|c|}{$11733 \cdot 5$} & \multicolumn{2}{|c|}{$12315 \cdot 3$} & \multicolumn{2}{|c|}{$11068 \cdot 9$} & \multicolumn{2}{|c|}{19934.9} \\
\hline
\end{tabular}

REML, restricted maximum likelihood; ref., referent category; FAS, Family Affluence Scale; SIMD, 2006 Scottish Index of Multiple Deprivation.

*Via restricted iterative generalised least squares (RIGLS).

tAIC is the Akaike Information Criterion; the larger this is, the worse the model fit.

$\$ 95 \% \mathrm{Cl}$ is above or below 0 . 
Table 4 Multilevel linear model for fruit consumption with interaction between rurality and deprivation (REML estimates*; SE) among 15-year-old adolescents; data from the 2010 Scotland Health Behaviour in School-aged Children survey

\begin{tabular}{|c|c|c|}
\hline & \multicolumn{2}{|c|}{ Fruit consumption } \\
\hline & Mean & SE \\
\hline \multicolumn{3}{|l|}{ Fixed effects } \\
\hline Constant/intercept & $5 \cdot 52$ & $2 \cdot 32$ \\
\hline Age & -0.09 & $0 \cdot 15$ \\
\hline \multicolumn{3}{|l|}{ Sex (ref.: Male) } \\
\hline Female & 0.42 & $0.09 \ddagger$ \\
\hline \multicolumn{3}{|l|}{ FAS (ref.: Low FAS) } \\
\hline Medium FAS & $0 \cdot 11$ & $0 \cdot 12$ \\
\hline High FAS & $0 \cdot 22$ & $0 \cdot 12$ \\
\hline Deprivation (SIMD score/10) & $-0 \cdot 17$ & $0.05 \ddagger$ \\
\hline \multicolumn{3}{|l|}{ School type (ref.: State school) } \\
\hline Independent school & 0.97 & $0 \cdot 29 \ddagger$ \\
\hline \multicolumn{3}{|l|}{ Rurality (ref.: Four Cities) } \\
\hline Other Urban & $-0 \cdot 11$ & $0 \cdot 24$ \\
\hline Accessible Towns & -0.30 & $0 \cdot 31$ \\
\hline Remote Towns & -0.49 & $0 \cdot 35$ \\
\hline Accessible Rural & $0 \cdot 70$ & $0 \cdot 32 \ddagger$ \\
\hline Remote Rural & $0 \cdot 70$ & $0.33 \ddagger$ \\
\hline \multicolumn{3}{|c|}{ Rurality $\times$ Deprivation interaction (ref.: Four Cities $\times$ SIMD score/10) } \\
\hline Other Urban $\times$ SIMD score/10 & -0.04 & 0.09 \\
\hline Accessible Towns $\times$ SIMD score $/ 10$ & -0.06 & $0 \cdot 15$ \\
\hline Remote Towns $\times$ SIMD score/10 & 0.21 & $0 \cdot 15$ \\
\hline Accessible Rural $\times$ SIMD score $/ 10$ & -0.44 & $0 \cdot 19 \ddagger$ \\
\hline Remote Rural $\times$ SIMD score/10 & -0.34 & $0 \cdot 17 \ddagger$ \\
\hline \multicolumn{3}{|l|}{ Random effects } \\
\hline Level 1 (child) variance & $5 \cdot 638$ & $0 \cdot 159$ \\
\hline Level 2 (school) variance & $0 \cdot 162$ & 0.057 \\
\hline Level 3 (education authority) variance & 0.000 & 0.000 \\
\hline-2 log likelihood & \multirow{3}{*}{\multicolumn{2}{|c|}{$\begin{array}{c}12189 \cdot 8 \\
20 \\
12229 \cdot 8\end{array}$}} \\
\hline No. of parameters & & \\
\hline $\mathrm{AIC}+$ & & \\
\hline
\end{tabular}

REML, restricted maximum likelihood; ref., referent category; FAS, Family Affluence Scale; SIMD, 2006 Scottish Index of Multiple Deprivation.

*Via restricted iterative generalised least squares (RIGLS).

tAIC is the Akaike Information Criterion; the larger this is, the worse the model fit.

$\ddagger 95 \% \mathrm{Cl}$ is above or below 0 .

schools ate fruit and vegetables more frequently (on approximately one more day per week), and chips and crisps less frequently, and had an average healthy eating score 5.78 greater than children from state-funded schools. Categorical FAS was significant for outcomes vegetable consumption and chips consumption and the healthy eating score. Area-level deprivation was also significant, independently of individual material wealth, for all but sweets consumption, with those with a greater score (i.e. more deprived) having a poorer diet.

After adjustment for sociodemographic variables, the rurality variable was significant for all but the fruit consumption models under the joint $\chi^{2}$ test, also supported by the AIC. Vegetables were consumed on significantly more days per week and sweets and crisps on significantly fewer days per week in Remote Rural areas than in Four Cities, after adjustment for all sociodemographic variables. This resulted in a healthy eating score on average 2.90 greater than in Four Cities. Accessible Rural areas also saw significantly lower sweets consumption than in Four Cities. Other Urban areas were the least healthy. When an interaction term was added between rurality and SIMD score/10, this was not significant for any of the consumption frequency outcomes other than fruit consumption. Socio-economic inequalities in fruit consumption were particularly great in Accessible Rural and Remote Rural areas, and significantly greater than those in urban areas (Table 4). Thus, those living in the most deprived areas of Remote Rural Scotland (SIMD score $=48 \cdot 87$ ) consumed fruit on approximately $1 \mathrm{~d}$ less per week than those living in Four Cities of equivalent deprivation. However, among the most affluent (SIMD = 3.03 ), Remote Rural residents ate fruit on average on $0.6 \mathrm{~d}$ more per week than their urban counterparts.

Rurality was less relevant in terms of eating behaviour (Table 5). For the outcome variable eating a family meal, the odds of sharing a family meal were particularly high among those living in Remote Towns. The odds of being on a diet were also higher in Remote Towns at the 94\% level of significance. However under the joint $\chi^{2}$ test the rurality variable was overall not significant for any of the eating behaviour outcomes.

Random effects showed that a large proportion of the variance for the consumption frequency models existed at the individual level (Table 3). Unexplained variance at the child level reduced but remained significant after adjustment for all explanatory variables. Variance at the school 
Table 5 Multilevel logistic models for categorical eating behaviour outcomes (MCMC* estimates; posterior SD, equivalent to SE) among 15-year-old adolescents; data from the 2010 Scotland Health Behaviour in School-aged Children survey

\begin{tabular}{|c|c|c|c|c|c|c|c|c|}
\hline & \multicolumn{2}{|c|}{ Meal together } & \multicolumn{2}{|c|}{ Regular breakfast } & \multicolumn{2}{|c|}{ On a diet } & \multicolumn{2}{|c|}{ Go to bed hungry } \\
\hline & Mean & SE & Mean & SE & Mean & $\mathrm{SE}$ & Mean & $\mathrm{SE}$ \\
\hline \multicolumn{9}{|l|}{ Fixed effects } \\
\hline Constant/intercept & $4 \cdot 67$ & $1 \cdot 36$ & $-2 \cdot 07$ & 1.43 & -1.09 & $1 \cdot 82$ & $-8 \cdot 48$ & $2 \cdot 90$ \\
\hline Age & -0.24 & 0.099 & 0.08 & 0.09 & -0.09 & $0 \cdot 12$ & $0 \cdot 37$ & $0 \cdot 18$ \\
\hline \multicolumn{9}{|l|}{ Sex (ref.: Male) } \\
\hline \multicolumn{9}{|l|}{ FAS (ref.: Low FAS) } \\
\hline Medium FAS & $0 \cdot 17$ & $0 \cdot 10$ & -0.05 & $0 \cdot 10$ & $0 \cdot 13$ & $0 \cdot 12$ & $-0 \cdot 41$ & $0 \cdot 23$ \\
\hline High FAS & 0.44 & $0.11 ब$ & -0.08 & $0 \cdot 11$ & 0.07 & $0 \cdot 13$ & -0.40 & $0 \cdot 23$ \\
\hline Deprivation (SIMD score/10) & $-0 \cdot 13$ & 0.03 & $0 \cdot 13$ & 0.04 & 0.08 & 0.049 & -0.05 & 0.08 \\
\hline \multicolumn{9}{|l|}{ School type (ref.: State school) } \\
\hline Independent school & 0.09 & 0.29 & $-0 \cdot 42$ & $0 \cdot 34$ & -0.51 & $0 \cdot 35$ & -0.56 & $0 \cdot 72$ \\
\hline \multicolumn{9}{|l|}{ Rurality (ref.: Four Cities) } \\
\hline Other Urban & -0.08 & $0 \cdot 15$ & $0 \cdot 11$ & $0 \cdot 16$ & 0.06 & $0 \cdot 16$ & $0 \cdot 14$ & $0 \cdot 31$ \\
\hline Accessible Towns & 0.07 & $0 \cdot 18$ & $0 \cdot 15$ & $0 \cdot 19$ & $0 \cdot 31$ & $0 \cdot 19$ & 0.45 & 0.35 \\
\hline Remote Towns & 0.45 & 0.209 & $0 \cdot 30$ & $0 \cdot 21$ & $0 \cdot 38$ & $0 \cdot 20$ & $0 \cdot 27$ & $0 \cdot 39$ \\
\hline Accessible Rural & -0.05 & $0 \cdot 16$ & -0.03 & $0 \cdot 16$ & -0.02 & $0 \cdot 18$ & $-0 \cdot 10$ & $0 \cdot 36$ \\
\hline Remote Rural & $0 \cdot 15$ & $0 \cdot 17$ & 0.20 & $0 \cdot 18$ & $0 \cdot 12$ & $0 \cdot 18$ & -0.08 & 0.35 \\
\hline \multicolumn{9}{|l|}{ Random effects } \\
\hline Level 1 (child) variancet & \multicolumn{2}{|c|}{1} & \multicolumn{2}{|c|}{1} & \multicolumn{2}{|c|}{1} & \multicolumn{2}{|c|}{1} \\
\hline Level 2 (school) variance & 0.028 & 0.030 & $0 \cdot 111$ & 0.054 & 0.014 & 0.020 & $0 \cdot 164$ & $0 \cdot 170$ \\
\hline Level 3 (education authority) variance & $0 \cdot 019$ & 0.022 & $0 \cdot 044$ & 0.044 & $0 \cdot 018$ & 0.022 & 0.054 & 0.073 \\
\hline $\bar{D} \ddagger$ & \multicolumn{2}{|c|}{$3152 \cdot 7$} & \multicolumn{2}{|c|}{3511.5} & \multicolumn{2}{|c|}{$2509 \cdot 5$} & \multicolumn{2}{|c|}{$988 \cdot 1$} \\
\hline$p_{\mathrm{D}} \S$ & \multirow{2}{*}{\multicolumn{2}{|c|}{$\begin{array}{r}29 \cdot 6 \\
3182 \cdot 3\end{array}$}} & \multirow{2}{*}{\multicolumn{2}{|c|}{$\begin{array}{r}64 \cdot 2 \\
3575 \cdot 6\end{array}$}} & \multirow{2}{*}{\multicolumn{2}{|c|}{$\begin{array}{r}20 \cdot 7 \\
2530 \cdot 2\end{array}$}} & \multirow{2}{*}{\multicolumn{2}{|c|}{$\begin{array}{r}29 \cdot 5 \\
1017 \cdot 6\end{array}$}} \\
\hline $\mathrm{DICl}$ & & & & & & & & \\
\hline
\end{tabular}

ref., referent category; FAS, Family Affluence Scale; SIMD, 2006 Scottish Index of Multiple Deprivation.

*Via Markov chain Monte Carlo (MCMC); estimates are based on a chain of length of 50000 following a burn-in of 5000

+Variance at the child level is constrained to 1.

$\ddagger \bar{D}$ is the expectation of the deviance and is a measure of how well the model fits the data.

$\S p_{\mathrm{D}}$ is the effective number of parameters.

IIDIC is the Deviance Information Criterion; the larger this is, the worse the model fit.

- $95 \% \mathrm{Cl}$ is above or below 1 .

level was significant for fruit and vegetable consumption. The random part of the breakfast consumption model also found significant unexplained variance at the school level (Table 5). The addition of a random slope at the school or education authority level to the rurality variable was not found to be significant for any of the models, suggesting that the relationship between rurality and consumption frequency did not vary by school or education authority.

\section{Discussion}

The present study suggests that young people from rural areas have a healthier diet than those living in urban areas. These differences exist even after adjustment for individual- and area-level deprivation. Adolescents living in Remote Rural Scotland have the highest consumption frequency of vegetables and the lowest consumption frequency of sweets and crisps. However, it is not the geography described by the classification Four Cities, but Other Urban areas (large towns of between 10000 and 125000 residents) that has the poorest diet.

Previously, urban-rural differences in adolescent food consumption were observed at the education authority level ${ }^{(11)}$. Those education authorities that included mostly rural areas, such as the Highlands and Islands, had a better diet. It was hypothesised that this was likely to be due to urban-rural differences in the historical tradition and culture of family meals, availability of food types and/or access to shops supplying snacks and convenience food, rather than strategic decision making by education authorities ${ }^{(11)}$. This is supported by the current study which shows that this geographic difference exists at the individual level of residence. However the current study does not support the theory relating to the family meal, as there was no difference in prevalence of family meals between Remote Rural Scotland and urban areas. Similarly, dieting behaviour, food poverty and breakfast consumption did not differ by rurality.

Previous work which examined availability and cost of fruit and vegetable items in Scotland found there to be no difference in food price by neighbourhood deprivation $^{(39)}$, with better access to grocery stores in more deprived areas and better availability in urban settings ${ }^{(40)}$. The current study found neighbourhood deprivation to be significant, independently of individuallevel material wealth, and associated with lower consumption frequency of fruit and vegetables and higher consumption frequency of crisps and chips. Again in contrast to work relating to availability of fruit and vegetables ${ }^{(40)}$, the relationship between neighbourhood 
deprivation and vegetable consumption did not differ by rurality in the current study; neighbourhood deprivation and rurality were associated with consumption independently of one another. For fruit consumption, associations with deprivation were more extreme in rural areas, suggesting greater socio-economic inequalities. However, Smith et al. ${ }^{(40)}$ found median times to stores, an inverse proxy for access, to be greatest for the most affluent residents of the (rural) islands of Scotland, indicating a reverse relationship. This suggests that in-store availability and access to food do not relate directly to adolescent consumption behaviour, at least for fruit and vegetables.

There may however be a relationship between in-store access and consumption of 'unhealthy foods'. Previously, modest correlations were seen between consumption of 'healthy' foods and between consumption of 'unhealthy' foods, but not between one another ${ }^{(11)}$, suggesting these two behaviours to be independent. High frequency of vegetable consumption in rural areas, for example, may be due to the ability to grow your own vegetables, while relatively low frequency of sweets and crisps consumption may be due to a scarcity of shops selling snacks on the way home from school. The reason for urban-rural differences in consumption frequency of foods might therefore depend on food type.

The role of schools in young people's diet, over and above rurality and deprivation, is highlighted in the current study. Of particular interest is the difference between state and independent schools. By age 15 years, the majority of independent schools in Scotland allow young people to leave the premises at lunchtime to buy their lunch. Even under these comparable circumstances, young people attending independent schools were significantly more likely to eat fruit and vegetables and less likely to eat crisps and chips. Variance at the school level remained significant for fruit and vegetable consumption, after adjustment for all factors, suggesting schools may have an important role to play in providing a healthy and tasty lunch menu. When rurality was allowed to vary at the school level (i.e. a random slope was introduced to the model), this was not significant for any of the outcomes, suggesting that the role of school is equally important across all ruralities. The school level was not significant for models of sweets and crisps consumption, probably due to the fact that schools are no longer allowed to sell these at snack or lunch times ${ }^{(3)}$. Although all schools are also encouraged to provide nutritious meals which include fruit and vegetables under the Schools (Nutrition and Health Promotion) Act (2007), quality and food choice are likely to vary. The impact of breakfast club provision in schools may also explain the significant variance at the school level for the outcome variable irregular breakfast consumption. In $2010,33 \%$ of primary and $58 \%$ of secondary schools in Scotland provided a breakfast club for pupils ${ }^{(41)}$. Again a random slope was not significant, suggesting a school effect on young people's diet across all of Scotland, regardless of rurality.

\section{Limitations and recommendations}

Although a minimum sample size of 350 per rurality was optimum, this was difficult to achieve with any precision because the boosted sample was selected by class rather than child's residence. As the FAS showed no sign of bias, the impact of not achieving this sample size for Accessible Towns and Remote Towns is unlikely to bias the results but may have resulted in an under-powering and therefore an overly conservative test of comparison between these ruralities and Four Cities within the models. Missing data may have resulted in a sample biased towards healthier children overall, although there is no reason to believe that this bias would vary by rurality and therefore should not bias urban-rural comparisons.

Another limitation of the study is that information about the diet of young people collected in the survey was incomplete, focusing only on five foods. The study is therefore limited to the food items described, acting as indicators of dietary intake rather than a comprehensive assessment of foods consumed. Furthermore, the indicators refer only to frequency of consumption and not to size or number of portions. It is possible, for example, that more than one portion of fruit is consumed on a single occasion (i.e. one time per week), therefore leading to underestimation of actual food intake. Nevertheless, the HBSC FFQ has been validated previously ${ }^{(27)}$ and, more generally, a recent review of FFQ among children and adolescents noted that highest validity was found when the questionnaire did not assess portion size, when it measured consumption over a relatively short time span (e.g. previous day/week) and when it was administered to young people rather than their parents $^{(42)}$. A $24 \mathrm{~h}$ recall method ${ }^{(43)}$, including portion size information and a more complete set of food groups, may instead be preferable in future studies. A computerised version of this method has also been developed for use with adolescent populations and across several European countries ${ }^{(44)}$.

Area measures of deprivation and rurality are at the 'data zone' small-area level (mean population size of 778 and range of between 500 and 1000), calculated in 2006 and 2008 respectively, and were assigned according to children's postcodes. These should therefore be reasonably accurate. Nevertheless, findings of urban-rural comparative studies are dependent on the definition of rurality ${ }^{(45)}$. This may therefore complicate or even invalidate comparisons with other studies using different measures of geography. Smith et al.'s(40) study measuring fruit and vegetable availability, referred to in the Discussion, used the same urban-rural classification as us and therefore should be approximately comparable.

Qualitative research is recommended to understand reasons for the urban-rural differences in food consumption 
reported in the current study. We have eliminated a few possible eating behaviours which might explain these differences, but further examination of why these differences exist is required. Future work is also recommended to look at differences in availability and access in relation to 'healthy' $v$. 'unhealthy' foods, as consumption of each is a separate independent behaviour. The impact of quality and choice of food available within schools should also be investigated.

\section{Acknowledgements}

Sources of funding: This work was supported by NHS Health Scotland and the Ludwig Boltzmann Institute. However, NHS Health Scotland and the Ludwig Boltzmann Institute had no role in the design, analysis or writing of this article. Conflicts of interest: There are no conflicts of interest. Author's contribution: K.L. conceived the idea for the study, carried out the analyses and wrote the manuscript. Acknowledgements: The Health Behaviour in School-aged Children (HBSC) study is an international survey conducted in collaboration with the WHO Regional Office for Europe. The author would like to acknowledge the HBSC international research network in forty-three countries that developed the study's research protocol.

\section{References}

1. World Health Organization (2004) Global Strategy on Diet, Physical Activity and Health. Geneva: WHO.

2. World Health Organization (2008) European Action Plan for Food and Nutrition Policy 2007-2012. Copenhagen: WHO Regional Office for Europe.

3. Scottish Executive (2007) Schools (Nutrition and Health Promotion) Act. Edinburgh: The Stationery Office.

4. Scottish Government (2008) Healthy Eating, Active Living: An Action Plan to Improve Diet, Increase Physical Activity and Tackle Obesity (2008-2011). Edinburgh: Scottish Government.

5. Scottish Government (2009) Recipe for Success - Scotland's National Food and Drink Policy. Edinburgh: Scottish Government.

6. Currie C, Levin K, Kirby J et al. (2011) Health Behaviour in School-Aged Children: World Health Organization Collaborative Cross-National Study (HBSC). Findings From the 2010 HBSC Survey in Scotland. Edinburgh: Child and Adolescent Health Research Unit, University of Edinburgh.

7. Leyland AH, Dundas R, McLoone P et al. (2007) Causespecific inequalities in mortality in Scotland: two decades of change. A population-based study. BMC Public Health 7, 172.

8. Scottish Government (2008) Equally Well Implementation Plan. Edinburgh: Scottish Government.

9. Levin KA \& Currie C (2009) Inequalities in toothbrushing among adolescents in Scotland 1998-2006. Health Educ Res 24, 87-97.

10. Levin KA, Currie C \& Muldoon J (2009) Mental well-being and subjective health of 11-15 year old boys and girls in Scotland, 1994-2006. Eur J Public Health 19, 605-610.

11. Levin KA, Kirby J, Currie C et al. (2012) Trends in adolescent eating behaviour: a multilevel cross-sectional study of 11-15 year olds in Scotland, 2002-2010. J Public Health 34, 523-531.
12. Barnett S, Roderick P, Martin D et al. (2001) A multilevel analysis of the effects of rurality and social deprivation on premature limiting long term illness. J Epidemiol Community Health 55, 44-51.

13. Levin KA \& Leyland AH (2006) A comparison of health inequalities in urban and rural Scotland. Soc Sci Med 62, $1457-1464$.

14. Levin KA \& Leyland AH (2005) Urban/rural inequalities in suicide in Scotland, 1981-1999. Soc Sci Med 60, 2877-2890.

15. Levin KA \& Leyland AH (2006) Urban-rural inequalities in ischaemic heart disease in Scotland, 1981-1999. Am J Public Health 96, 145-151.

16. Weiss SJ, Ellis R, Ernst AA et al. (2001) A comparison of rural and urban ambulance crashes. Am J Emerg Med 19, 52-56.

17. Campbell NC, Elliott AM, Sharp L et al. (2001) Rural and urban differences in stage at diagnosis of colorectal and lung cancers. Br J Cancer 84, 910-914.

18. Pearce J, Witten K \& Bartie P (2006) Neighbourhoods and health: a GIS approach to measuring community resource accessibility. J Epidemiol Community Health 60, 389-395.

19. Veitch C (2009) Impact of rurality on environmental determinants and hazards. Aust J Rural Health 17, 16-20.

20. Bishop J, Clark D, Harris V et al. (2004) Deprivation and Urban Rural Measurement in ISD. Edinburgh: NHS ISD; available at http://www.isdscotland.org/isd/files/Measuring_ deprivation_in_ISD_v3.pdf

21. Schulz A \& Northridge ME (2004) Social determinants of health: implications for environmental health promotion. Health Educ Behav 31, 455-471.

22. Wagstaff A (1991) QALYs and the equity-efficiency trade-off. $J$ Health Econ 10, 21-41.

23. Leyland $\mathrm{AH}$ (2004) Increasing inequalities in premature mortality in Great Britain. J Epidemiol Community Health 58, 296-302.

24. Scottish Executive Health Department (2003) Inequalities in Health. Report of the Measuring Inequalities in Health Working Group. Edinburgh: Scottish Executive.

25. Currie C, Grieber R, Inchley J et al. (2010) Health Behaviour in School-Aged Children (HBSC) Study Protocol: Background, Methodology and Mandatory Items for the 2009/10 Survey. Edinburgh: University of Edinburgh.

26. Currie C, Levin K \& Todd J (2008) Health Behaviour in School-aged Children: World Health Organization Collaborative Cross-National Study (HBSC). Findings from the 2006 HBSC survey in Scotland. Edinburgh: University of Edinburgh; available at http://www.st-andrews.ac. uk/cahru/publications/reports_downloads/HBSC_National_ Report_2008.pdf

27. Vereecken CA \& Maes L (2003) A Belgian study on the reliability and relative validity of the Health Behaviour in School-Aged Children food-frequency questionnaire. Public Health Nutr 6, 581-588.

28. Vereecken CA, de Henauw S \& Maes L (2005) Adolescents' food habits: results of the Health Behaviour in School-aged Children survey. Br J Nutr 94, 423-431.

29. Vereecken CA, Rossi S, Giacchi MV et al. (2008) Comparison of a short food-frequency questionnaire and derived indices with a seven-day diet record in Belgian and Italian children. Int J Public Health 53, 297-305.

30. Vereecken CA, Haerens L, De Bourdeaudhuij I et al. (2010) The relationship between children's home food environment and dietary patterns in childhood and adolescence. Public Health Nutr 13, 1729-1735.

31. Molcho M, Nic Gabhainn S, Kelly C et al. (2007) Food poverty and health among schoolchildren in Ireland: findings from the Health Behaviour in School-aged Children (HBSC) study. Public Health Nutr 10, 364-370.

32. Currie C, Zanotti C, Morgan A et al. (2012) Social Determinants of Health and Well-Being Among Young 
People. Health Behaviour in School-Aged Children (HBSC) Study: International Report from the 2009/2010 Survey. Health Policy for Children and Adolescents no. 6. Copenhagen: WHO Regional Office for Europe.

33. Currie C, Molcho M, Boyce W et al. (2008) Researching health inequalities in adolescents: the development of the Health Behaviour in School-aged Children (HBSC) Family Affluence Scale. Soc Sci Med 66, 1429-1436.

34. Scottish Executive (2006) Scottish Index of Multiple Deprivation 2006: General Report. Edinburgh: Scottish Executive; available at http://www.scotland.gov.uk/Resource/ Doc/151578/0040731.pdf

35. Scottish Government (2008) Scottish Government Urban Rural Classification 2007-2008. Edinburgh: Scottish Government; available at http://www.scotland.gov.uk/Publications/ 2008/07/29152642/0

36. Levin KA, Davies CA, Douglas GVA et al. (2010) Urban-rural differences in dental caries of 5-year old children in Scotland. Soc Sci Med 71, 2020-2027.

37. Rasbash J, Charlton C, Browne WJ et al. (2005) MLwiN version 2.02. Bristol: University of Bristol.

38. Spiegelhalter DJ, Best NG, Carlin BR et al. (2002) Bayesian measures of model complexity and fit (with discussion). J R Stat Soc Series B Stat Methodol 64, 583-639.

39. Cummins S, Smith DM, Aitken $Z$ et al. (2010) Neighbourhood deprivation and the price and availability of fruit and vegetables in Scotland. J Hum Nutr Diet 23, 494-501.

40. Smith DM, Cummins S, Taylor M et al. (2010) Neighbourhood food environment and area deprivation: spatial accessibility to grocery stores selling fresh fruit and vegetables in urban and rural settings. Int J Epidemiol 39, 277-284.

41. Scottish Government (2010) A National Statistics Publication for Scotland: School Meals in Scotland, 2010. Edinburgh: The Stationery Office; available at http://www. scotland.gov.uk/Publications/2010/07/06095048/0

42. Kolodziejczyk JK, Merchant G \& Norman GJ (2012) Reliability and validity of child/adolescent food frequency questionnaires that assess foods and/or food groups. J Pediatr Gastroenterol Nutr 55, 4-13.

43. Vucic V, Glibetic M, Novakovic R et al. (2009) Dietary assessment methods used for low-income populations in food consumption surveys: a literature review. Br J Nutr 101, Suppl. 2, S95-S101.

44. Vereecken CA, Covents $\mathrm{M}$, Sichert-Hellert W et al. (2008) Development and evaluation of a self-administered computerized 24-h dietary recall method for adolescents in Europe. Int J Obes (Lond) 32, Suppl. 5, S26-S34.

45. Brady JE \& Weitzman BC (2007) Inconsistencies in place definition: how different operational place definitions affect estimates of adolescent smoking and drinking risk. Health Place 13, 562-568. 